\title{
Erratum to: Supporting Management of Hybrid OSS Communities - A Stakeholder Analysis Approach
}

\author{
Hanna Mäenpää ${ }^{1(凶)}$, Tero Kojo ${ }^{2}$, Myriam Munezero ${ }^{1}$, \\ Fabian Fagerholm ${ }^{1}$, Terhi Kilamo ${ }^{3}$, Mikko Nurminen ${ }^{3}$, \\ and Tomi Männistö ${ }^{1}$ \\ ${ }^{1}$ University of Helsinki, Helsinki, Finland \\ \{hanna. maenpaa, myriam. munezero, fabian. fagerholm, \\ tomi.mannisto\}@cs.helsinki.fi \\ 2 The Qt Company, Espoo, Finland \\ tero.kojo@qt.io \\ 3 Tampere Technical University, Tampere, Finland \\ \{terhi.kilamo,mikko.nurminen\}@tut.fi
}

\section{Erratum to:}

Chapter 7 in: P. Abrahamsson et al. (Eds.)

Product-Focused Software Process Improvement

DOI: 10.1007/978-3-319-49094-6_7

In the paper starting on page 102 of this volume, the word "communities" was spelled incorrectly in the main title. It has to read "Communities" instead of "Communnities".

The updated original online version for this chapter can be found at DOI: 10.1007/978-3-319-49094-6_7 